\title{
Applying multiple land surface temperature products to derive heat fluxes over a grassland site
}

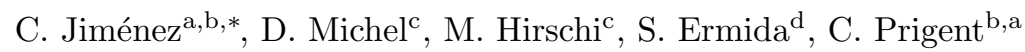 \\ ${ }^{a}$ Estellus, Paris, France \\ ${ }^{b}$ LERMA, Paris Observatory, Paris, France \\ ${ }^{c}$ Institute for Atmospheric and Climate Science, ETH Zürich, Zürich, Switzerland \\ ${ }^{d}$ Instituto Dom Luiz, University of Lisbon, Portugal
}

\begin{abstract}
Land heat fluxes are essential components of the water and energy cycle and important variables in the management of agronomy and forestry resources. The estimation of the heat fluxes can be done with a number of methodologies, with some of them having the land surface temperature $(T s)$ as one of their key inputs to derive the fluxes. Here the production of $T s$-driven surface heat fluxes over a grassland site in Switzerland is demonstrated by running a specific heat flux methodology (SEBS) fed by a number of satellite $T s$ estimates (from the instruments AATSR, MODIS, SEVIRI, AMSR-E, and SSMIS). The Ts estimates are compared with an in situ estimate derived from radiometric observations at the station, and the satellite latent heat flux $(L E)$ estimates with the station Eddy Covariance (EC) measurements. The satellite $T s$ products include estimates at different spatial resolutions (from $\sim 1$ to $\sim 25 \mathrm{~km}$ ) and time samplings (from 2 overpasses per day to $1 / 2$ hourly observations). Root Mean Square Differences (RMSDs) between the daytime satellite and station $T s$ are 2.72 (AATSR), 4.41 (MODIS), 3.59 (SEVIRI), 3.81 (AMSR-E), and 2.79 (SSMIS), but given the different time samplings and spatial resolutions its is difficult to be conclusive about the accuracy of the $T s$ estimates. Concerning the flux estimates, for those sensors with midday overpasses, a RMSD of $\sim 25 \%$ are found when comparing the instantaneous latent flux ( $L E$, or evaporation expressed as an amount of
\end{abstract}

*corresponding author: carlos.jimenez@estellus.fr

Preprint submitted to Journal of ${ }^{A} T_{E} X$ Templates

January 21, 2017

(C) 2016. This manuscript version is made available under the Elsevier user license http://www.elsevier.com/open-access/userlicense/1.0/ 
water) at satellite overpass with the EC observations, which compares well with the accuracy reported elsewhere for similar landscapes. Given that both $T s$ and $L E$ are evaluated at the station, a link between $T s$ and $L E$ accuracy is investigated, but its is not apparent for this specific comparison. This could be related to SEBS accuracy also depending on other variables, apart from $T s$, but also to the representativeness of the metrics used for the evaluation given the spatial miss-matches existing between the satellite estimates and station observations. Discrepancies were observed between the EC fluxes, the measured surface available energy, and the evaporation estimates from a lysimeter also present at the station, illustrating also the difficulties of the ground observations to provide accurate heat fluxes for satellite evaluation.

Keywords: Land surface temperature; surface heat fluxes; terrestrial evaporation; grasslands; satellite remote sensing.

\section{Introduction}

Land heat fluxes govern the interactions between the Earth surface and the atmosphere [e.g., 1], are essential components of the water and energy cycles [e.g., 2], and play a key role in the climate system [e.g., 3] . The latent heat

5 flux is also an important variable in the management of agronomy, forestry, and hydrological resources and evaporation estimates (the heat flux expressed as an amount of water) at different spatial scales, from individual plants for managing irrigation to basin scales to evaluate water resources, are required in many applications [e.g. 4, 5,6].

In situ measurements of land heat fluxes are operated during field experiments [e.g. 7] and by some flux tower networks [e.g 8]. Being point measurements and requiring special equipment, they cannot be applied for routine measurements covering large areas. Therefore, more readily available observations are combined with well known flux formulations [e.g., 9, 10] to obtain local estimates. For instance, the Food and Agriculture Organization (FAO) recommends the Penman-Monteith method to estimate crops evaporation, which 
requires a number of surface meteorological inputs and relevant crop coefficients [11]. Satellite remote sensing could be used for routine estimates at different time and spatial scales, but the challenge here is that fluxes do not have a direct signature that can be remotely detected, so again satellite observations related to surface temperature, soil moisture, or vegetation need to be combined to infer the fluxes. An overview of different methodologies for $L E$ estimation can be found in [3].

If satellite surface-derived skin temperature $(T s)$ is available, in principle bulk transfer calculations from the aerodynamic theory of turbulent transfer can be used to produce heat fluxes [e.g., 12]. Ts is used as a proxy for the aerodynamic surface temperature (Tas), and the sensible heat flux $(H)$ is derived from the difference between the skin temperature $T s$ and a measured air temperature $(T a)$, scaled by a transfer coefficient characterizing the transport 30 of heat and depending on site-specific data, such as surface roughness and wind speed. The latent heat flux $(L E)$ can then be estimated from an energy balance model, assuming the surface radiation and the ground flux are known.

Given the routine availability of $T s$ from satellite sensors, estimating heat fluxes from satellite-derived $T s$ is a common practice (see e.g. [13] for a review).

35 However, it is not without challenges. Difficulties first arise from the fact that differences between $T s$ and $T a$ are rather limited for many environmental conditions and the accuracy of each measurement (often of the same order of their difference) can have a detrimental effect on the estimated $T s$. In addition, the difference between Tas and Ts can be significant [e.g., 14], as well as the difference between the soil and the vegetation temperatures with only one integrated temperature being estimated from the satellite instrument [e.g., 15, 16]. Still based on the bulk formulation, more elaborated models try to overcome some of these difficulties by partitioning the measured $T s$ into its soil and canopy components, and by exploiting the relationship between soil moisture and ther45 mal inertia. For instance, formulations have been derived to constrain the heat fluxes with the diurnal gradient of $T s$ [e.g., 17, 18], or with the dry-wet limiting conditions imposed by the surface energy balance [e.g., 19]. Other schemes use 
relationships between $T s$ and other satellite observations, such as the vegetation index [e.g., 20, 16], and as they typically require local calibrations, there are better suited for field scale applications (e.g., for irrigation management) rather than for relatively large scales (e.g. to derive basin integrated estimates).

Here we demonstrate the production of $T s$-driven surface heat fluxes over a grassland site in Switzerland. The European Space Agency (ESA) is currently running the GlobTemperature Data User Element (DUE) project (www . globtemperature.info) aiming to provide high quality $T s$ data and facilitate wider use of global-scale satellite $T s$ in research and operational user communities. The present study serves to illustrate the potential for using satellite Ts to derive land heat fluxes from an end user point of view. In that regard, the main objectives of the study are: (1) to select and cross-compare a range of 60 Ts products over a specific location, (2) to derive heat fluxes with the available $T s$ products, (3) to discuss the impact of $T s$ product selection on the derived fluxes, and (4) to highlight the difficulties of evaluating satellite $T s$ and heat fluxes. The location selected for the exercise is a grassland site situated at the Rietholzbach Research Catchment in Switzerland, where in situ observations enable comparisons between the satellite $T s$ products and derived heat fluxes with ground measurements. The Ts products include datasets from the Spinning Enhanced Visible and Infrared Imager (SEVIRI) onboard the geostationary satellite Meteosat Second Generation (MSG)-2, the Advanced Along-Track Scanning Radiometer (AATSR) onboard ESA EnviSAT polar-orbiting satellite, the Moderate Resolution Imaging Spectroradiometer (MODIS) onboard the Terra and Aqua satellites, the Special Sensor Microwave Imager/Sounder (SSMIS) onboard the United States Air Force Defense Meteorological Satellite Program (DMSP) satellites, and the Advanced Microwave Scanning Radiometer - Earth observing system (AMSR-E) on board the Aqua satellite. Finally, con75 cerning the heat flux methodology, the Surface Energy Balance System (SEBS) model [19] will be applied. SEBS is an energy balance model based on a detailed parameterization of $H$ at the surface, with $L E$ derived from the surface energy balance once $H$ is estimated. It is a one-source model, i.e., bulk fluxes 
are derived without partitioning the soil and canopy contributions, but this is not judged as a severe limitation given the vegetation coverage of the selected location. In the past, SEBS has proven to estimate realistic evaporation rates at a variety of scales, ranging from local to regional [e.g. 21, 22, 23], although difficulties connected to the $T s$ uncertainty and the derivation of the heat transfer coefficient have also been reported [e.g. 24, 25, 26].

The paper is organized as follows: first, the study site and its ground observations are presented, followed by a description of the heat flux model and the inputs required to run the model, including the different $T s$ products. A comparison of the different $T s$ products and the derived heat fluxes is then made, with a discussion about the impact of product selection in the derived fluxes.

90 Finally, the main conclusions of the study are summarized.

\section{Methods and data}

\subsection{Site description}

The study is conducted at the hydrometeorological research catchment Rietholzbach in northeastern Switzerland $\left[47.37^{\circ} \mathrm{N}, 8.99^{\circ} \mathrm{E}, 795 \mathrm{~m}\right.$ asl; see 27 , and www.iac.ethz.ch/url/rietholzbach for an overview of the site]. The prealpine catchment (elevation range: $682-950 \mathrm{~m}$ asl) drains an area of $3.31 \mathrm{~km}^{2}$ and is a headwater catchment of the Thur river. The local conditions are characterized by a temperate humid climate with mean air temperature of $7.0^{\circ} \mathrm{C}$ and a mean annual precipitation amount of $1447 \mathrm{~mm}$. The land use in the catchment consists of a mixture of grasslands and forest (73\% grasslands, $24 \%$ forest and trees, the rest sparse settlements).

The measurements considered in this article are conducted at the research site "Büel", which is located in the upper part of the Rietholzbach catchment within a grassland area. The on-going measurements include standard meteorological and hydrological variables such as air temperature, precipitation, air humidity, radiation, soil moisture, runoff, and ground water level. Evapotranspiration measurements are provided by a lysimeter and an eddy covariance 
(EC) flux tower [see 28].

The installed weighing lysimeter allows the quantitative measurement of water mass changes within a soil column using three load cells. In combination with the measurement of precipitation and lysimeter seepage (both measured using tipping buckets), actual evapotranspiration is estimated on an hourly time scale [28]. The lysimeter at "Büel" has a radius of $1 \mathrm{~m}$ and a total depth of $2.5 \mathrm{~m}$ including a gravel filter layer at the bottom. The surface is covered by grass and maintained in agreement with the surrounding grassland area (with a specified cutting scheme and fertilization).

The EC measurements (i.e., sensible and latent heat fluxes, $H$ and $L E$ ) used here are conducted $2 \mathrm{~m}$ above ground at a flux tower located in $\sim 10 \mathrm{~m}$ distance from the lysimeter. The tower is equipped with an ultrasonic anemometer ${ }_{120}$ (CSAT3, Campbell Scientific Inc., USA) as well as an open-path $\mathrm{CO}_{2} / \mathrm{H}_{2} \mathrm{O}$ infrared gas analyser (Li-7500, LI-COR Biosciences, USA). The instruments are operated at $10 \mathrm{~Hz}$ and fluxes are calculated at a resolution of 30 minutes and aggregated to hourly output.

Further measurements used in this study include precipitation $P$ (measured with Joss-Tognini type, Lambrecht, Germany), net radiation Rn (CM21 and CG4, Kipp\&Zonen, The Netherlands), ground heat flux G (HFP01, Hukseflux, The Netherlands), air temperature Ta (Thygan, Meteolabor, Switzerland) and wind velocity $u$ (R. M. Young, USA). All these variables are available at 5minute resolution, except for $G$ measured at hourly resolution.

130 2.2. $S E B S$

SEBS [19] is a one-source energy balance algorithm that is arguably one of the most widely used energy balance approaches to derive heat fluxes. The SEBS model calculates the sensible heat flux $(H)$ based on the Monin and Obukhov theory [29]: 


$$
\begin{array}{r}
\theta_{0}-\theta_{\mathrm{a}}=\frac{H}{k u_{*} \rho c_{p}}\left[\ln \left(\frac{z-d_{0}}{z_{0 \mathrm{~h}}}\right)-\Gamma_{\mathrm{h}}\left(\frac{z-d_{0}}{L}\right)+\Gamma_{\mathrm{h}}\left(\frac{z_{0 \mathrm{~h}}}{L}\right)\right] \\
u=\frac{u_{*}}{k}\left[\ln \left(\frac{z-d_{0}}{z_{0 \mathrm{~m}}}\right)-\Gamma_{\mathrm{m}}\left(\frac{z-d_{0}}{L}\right)+\Gamma_{\mathrm{m}}\left(\frac{z_{0 \mathrm{~h}}}{L}\right)\right] \\
L=-\frac{\rho c_{p} u_{*}^{3} \theta_{\mathrm{v}}}{k g H},
\end{array}
$$

\subsection{SEBS Inputs}

The satellite $T s$ products considered in the study are listed in Table 1. They include two infrared instruments onboard polar satellites (AATSR and MODIS), one infared instrument onboard a geostationary satellite (SEVIRI), 
and two microwave instruments onboard polar satellites (SSMIS and AMSRE). The AATSR Ts estimates come from the GlobTemperature harmonised dataset, reformatted from [30]. SEVIRI and MODIS estimates are extracted from the original products [31,32]. The SSMIS and AMSR-E estimates have been processed from a data record of SSMIS [33] and AMSR-E [34] brightness temperatures following the methodology of [35] using databases of SSM/I [36] and AMSR-E [37] radiances and retrieved Ts. The AMSR-E Ts can be accessed from the GlobTemperature archive, while the SSMIS estimates are not publicly available yet but can be accessed upon request to the authors.

The spatial resolutions of the $T s$ estimates range from the $\sim 1 \mathrm{~km}$ of AATSR and MODIS to the $\sim 25 \mathrm{~km}$ of SSMIS. Temporal sampling ranges from the 170 AATSR 3-day revisi time, one day and night overpass, to the daily 15 minutes sampling of SEVIRI. Notice that the infrared instruments cannot provide $T s$ estimates under cloudy conditions, and that the $T s$ estimates provided by the microwave instruments correspond to a depth-integrated temperature that can deviates from the infrared skin temperature on some occasions (e.g., for very dry soils where the microwave penetration depth can be of several centimeters).

Ground $T s$ is derived from inverting the equation relating surface temperature and the upwelling and downwelling broadband radiance measured by the station longwave radiometer. Broadband emissivity is required and it is estimated from the MODIS-based Global Infrared Land Surface Emissivity Database [38] operated by the Cooperative Institute for Meteorological Satellite Studies (CIMSS). The Ts estimates are calculated by following the approach suggested by [39] using a linear combination of narrowband emissivities at 8.5, 11 , and $12 \mu \mathrm{m}$.

\subsubsection{Surface Meteorology}

Apart from $T s$, SEBS also requires estimates for the surface meteorological variables $R n, G, T a$, and $u$. Here we use the station ground to represent as closely as possible the station conditions, but a typical larger scale application will require spatially distributed values of those parameters at adequate 
Table 1: Table summarizing the Ts products. Listed are the sensor name, the satellite platform, the approximated spatial resolution, the approximated equatorial overpass local time (descending node), the product name, and the data archive.

\begin{tabular}{llllll}
\hline Sensor & Platform & Spatial & Overpass & Product & Archive \\
\hline \hline AATSR & Envisat & $\sim 1 \mathrm{~km}$ & $\sim 10.00$ & GT_SSD-L2-AATSR & GlobTemperature \\
& & & & & \\
\hline MODIS & Aqua & $\sim 1 \mathrm{~km}$ & $\sim 1.30$ & MYD11A1 v5 & \\
& Terra & & $\sim 10.30$ & MOD11A1 v5 & LP DAAC \\
& & & & & \\
\hline SEVIRI & MSG-2 & $\sim 4 \mathrm{~km}$ & every & GT_SSD-L2-SEVIR & LSA SAF \\
& & & 15 min & & \\
\hline AMSR-E & Aqua & $\sim 15 \mathrm{~km}$ & $\sim 1.30$ & GT_SSD-L2-AMSRE & GlobTemperature \\
& & & & \\
\hline SSMIS & F16 & $\sim 25 \mathrm{~km}$ & $\sim 7.00$ & & \\
& F17 & & $\sim 5.30$ & & Estellus * \\
& F18 & & $\sim 8.00$ & LST_SSMIS_v1 & \\
\hline
\end{tabular}

* Not publically available.

spatial and time resolutions. While satellite-based estimates exist for $R n$ (although typically at coarse spatial resolution, e.g., the Surface Radiation Budget product [40]), $G$ cannot be measured from space (it is approximated by some relationships to other radiative fluxes, e.g., Equation 3 in [19]). $T a$ is challenging to retrieve from satellite observations (but some products exist, e.g., see an evaluation of the AIRS Ta and near-surface humidity in [41]), and $u$ over land cannot currently be provided from satellite instruments. Alternatives are to use products based on extrapolating the synoptic network of weather stations to regular spatial grids (although normally at coarse spatial and temporal resolutions, e.g., the Climate Research Unit (CRU) monthly time series dataset [42]), or the outputs of Numerical Weather Prediction forecasts or reanalyses 200 (with better temporal resolutions, but further away from a "purely" observational product). For this study the $\sim 7 \mathrm{~km}$ hourly analysis fields of the weather 
forecasting model Consortium for Small-Scale Modelling (COSMO) run by MeteoSwiss are used to obtain a second $T a$ product and a daily estimate of the height of the planetary boundary layer (required for the calculation of the SEBS stability correction functions).

\subsubsection{Vegetation}

SEBS requires the one-side Leaf Area Index (LAI) to derive a within-canopy wind speed profile extinction coefficient as part of the calculations for the scalar roughness lengths (Equation 7 in [19]). Here we use the MODIS product MOD15A1 (version 5) to provide $1 \mathrm{~km}$ LAI over the station.

Vegetation height is also required, its is approximated by a $15 \mathrm{~cm}$ height constant throughout the year (the grass at the station is regularly mowed). The fraction of vegetation cover (FVC) is also used by SEBS to weight the contributions of the estimated soil and canopy land fractions to the scalar roughness for heat transfer $z_{0 \mathrm{~h}}$ (see Equation 9 in [19]). In the absence of a better estimate and given that the observation tower fetch consists of a uniformly thick layer of grass plants, we approximate the FVC to one (i.e, we consider the contribution of the heat fluxes coming from the grass plants much more significant than any contribution from the soil).

\subsection{Data processing}

Most in situ data relevant to this study is available every 5 minutes, but this excludes the heat and ground fluxes (there are available every hour). A time miss-match can be critical for the comparison of the satellite $T s$ with the station $T s$ at the times of the day with strong temperature gradients (e.g., at the mid-morning AATSR and MODIS (Aqua) overpasses). Therefore, the original 5 minutes resolution has been retained (as opposed to integrating all data to hourly resolution), with the heat fluxes re-sampled at 5 minutes resolution by assuming a constant evaporative fraction (ratio of $L E$ to $R n$ ) during the hourly interval, and the ground flux constant during the hourly interval. 
Satellite overpasses are selected when the station location is within the satellite footprint. Given the different satellite footprint resolutions (see Table 1), the representativness of the satellite footprint with respect to the station will depend on the sensor. Within a radius of $\sim 100 \mathrm{~m}$ of the station tower the land cover is purely grassland. Away from the tower the landscape is predominantly a mixture of grasslands and forest (for the $\sim 3 \mathrm{~km}^{2}$ research catchment the proportion is $73 \%$ grasslands, $24 \%$ forest and trees, and the rest sparse settlements). Therefore, even at the $\sim 1 \mathrm{~km}$ resolution of AATSR and MODIS the satellite footprint cannot be considered as purely grassland, and some of the discrepancies between the different $T s$ and flux estimates can be attributed to the different percentages of grassland and forest sampled by each instrument. Regarding the time matching between satellite overpass and ground data, given that the ground data is available every 5 minutes, a maximum time miss-match will be 2.5 minutes.

Cloud contamination (i.e., the satellite product cloud mask wrongly identifies a cloudy pixel as clear-sky) can occur in the infrared $T s$ estimates. To reduce the risk of having those pixels as part of the comparisons, the $\sim 5 \mathrm{~km}$ AATSR (GT_SSD-L3-AATSR) and MODIS (MOD11C1) products have also been inspected, and only those $1 \mathrm{~km} T s$ where the corresponding $5 \mathrm{~km} T s$ scene was mainly composed of clear pixels are kept (at least $85 \%$ of clear pixels). This strategy cannot be applied to SEVIRI (already at a resolution of $\sim 5 \mathrm{~km}$ ), but a much colder $T s$ with respect to the ground estimate (difference larger than $15 \mathrm{~K}$ ) has been imposed as a criterium to assure that obvious cloud contaminated pixels are not included in the comparisons. The microwave $T s$ estimate do not suffer in general from cloudiness (although strong convection with heavy precipitating cells can affect the $T s$ retrievals).

Given the lack of specific parameterisations in SEBS to deal with negative evaporation (i.e., condensation, typically occurring at night) or evaporation from snow surfaces (i.e., sublimation), and the difficulties of the EC instruments to measure the fluxes in situations associated with low turbulence and precipitation conditions, the study will only consider daytime $T s, L E$, and $H$ for snow and 
rain-free conditions. To detect snow a flag based on the observed shortwave albedo is used to filter the data. Nighttime fluxes are typically small (compared with daytime fluxes) and in principle for applications related to agriculture and water management the daytime fluxes suffice. Nevertheless, nightime and snow fluxes can be of importance for some climatological applications [e.g. 43].

\section{Results and discussion}

\subsection{Temperature and fluxes at the station}

Equation 1 shows the large dependence of SEBS derivation of $H$ on the difference of the potential temperatures between the surface and the overlaying correspond to the summer months where the available energy is the largest. The largest evaporative fractions (estimated as the EC $L E /(H+L E)$ ) also occur in the summer months and can reach values close to 0.8 , indicating that the region 

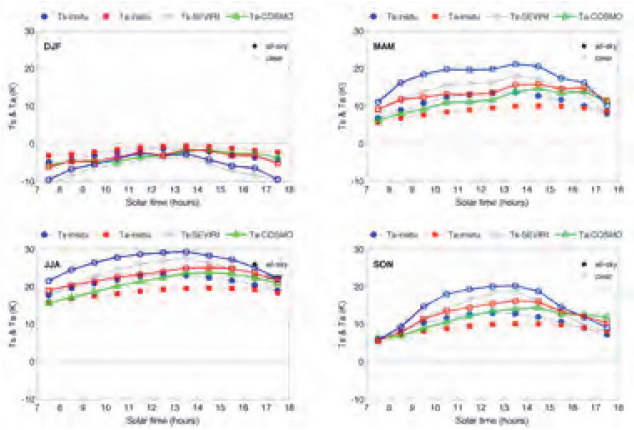

Figure 1: Seasonal skin temperature $(T s)$ and air temperature $(T a)$ cycles (top-left for winter, top-right for spring, bottom-left for summer, bottom-right for autumn). The data shows the station all-sky and clear-sky Ts (blue squares) and Ta (red dots), and the clear-sky SEVIRI Ts (black triangles) and COSMO Ta (green triangles). Closed symbols correspond to the all-sky measurements at the station, opened symbols correspond clear-sky measurements only (by applying SEVIRI cloud mask).

is not water limited in summer and that the available energy controls to a large extent the heat fluxes. In terms of energy closure, the averaged seasonal sum of $L E$ and $H$ is in most cases smaller than the observed $R n-G$, although the difference tends to get smaller in the late afternoon hours. Overall, the average daytime closure ratio estimated at the site (i.e., the total amount of the daytime heat fluxes to the daytime available energy) amounts to $88 \%$ [28]. Regarding the agreement of the EC and lysimeter $L E$, for this year the largest disagreement happens in summer, where the seasonally averaged lysimeter $L E$ can be up to $20 \%$ larger than the EC $L E$ at midday. The agreement is closer in spring and autumn, while in winter the very low $L E$ results in small absolute differences (but large in relative terms) [see also 28].

\subsection{Satellite surface temperature time sampling and spatial resolution}

The time sampling and spatial resolution required in a heat flux product depends on the application. One value per day should be enough for most agriculture and forest applications, though it may not be enough for a climatological application that requires resolving the diurnal cycle. Similar considerations 

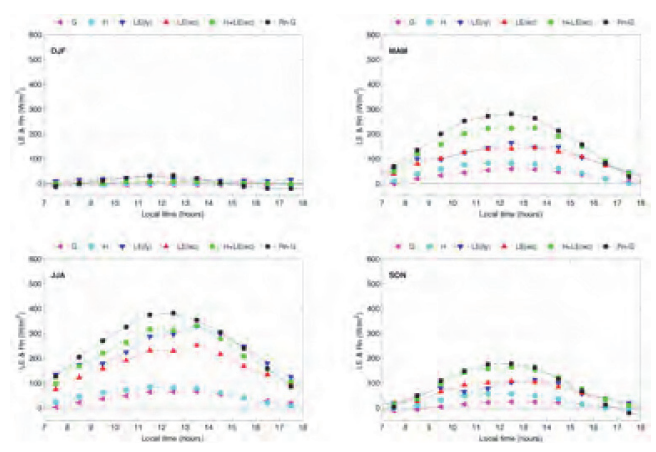

Figure 2: Seasonal cycles for the ground heat flux ( $G$, magenta triangles), the sensible heat flux ( $H$, cyan circles), and latnet heat flux ( $L E$, blue triangles for the lysimeter, red triangles for the EC instrument) observed at the station (top-left for winter, top-right for spring, bottomleft for summer, bottom-right for autumn). The sum of the surface heat fluxes measured by the EC instruments $(H+L E$, green squares) and the surface available energy (difference between the surface net radiation $(R n)$ and $G$, black squares) are also plotted.

apply to the required time continuity of the data record. While a few flux estimates per month may be enough as an indicator of forest health (in this case the missing data because of cloud coverage will not be critical), a climatological application may demand continuity in time (wich can be provided by the "allweather" Ts from instruments such as SSMIS). Regarding spatial resolution, this is also very much application dependent, and while the resolution of the microwave instrument AMSR-E and SSMIS ( $\sim 15$ and $\sim 25 \mathrm{~km}$, respectively) can be adequate for a global climatological data record, it will not be of much utility to spatially resolve evaporation for most crops. For these applications the much finer resolution of instruments such as AATSR and MODIS is more adequate. However, it should be mentioned that even their $1 \mathrm{~km}$ resolution can be considered too coarse for many agriculture, forestry, and land management applications, and tens of meters is a more desirable resolution [e.g. 17]. Instruments with finer resolutions in thermal infrared bands such as the Advanced Spaceborne Thermal Emission and Reflection Radiometer (ASTER, $90 \mathrm{~m}$ resolution) could be more relevant for those applications (e.g., see an evaluation of 


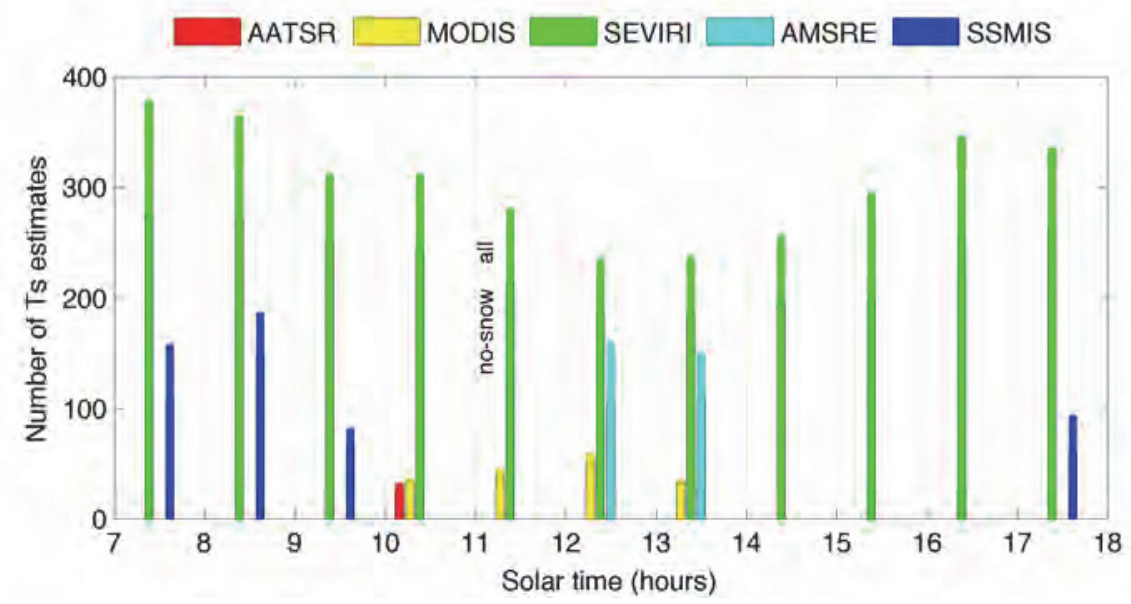

Figure 3: Number of satellite daylight overpasses with an available skin temperature $(T s)$ estimate at site in 2010. The number is given for all overpasses (full bar) and for the overpasses without snow on the ground (black outlined bar over-plotted, indicated with text for the 11 hour data).

SEBS with ASTER imagery at [44]), although their typically very long revisiting time (16 days for ASTER) can also be a severe limitation depending on application. For even finer resolutions and coverage during overcast conditions, thermal sensors is becoming an attractive possibility [e.g. 45].

Figure 3 illustrates the time availability of satellite $T s$ for the specific location of the study (after imposing the time and space matching criteria discussed in Section 2.4). The snow-free and total number of available $T s$ estimates is given in the figure. AATSR overpasses with usable $T s$ estimates are very limited in number due to the 3-days revisiting time, frequent cloudiness at the station location, and the constraint of having contiguous clear-sky pixels to minimize cloud contamination (see Section 2.4). MODIS is also affected by cloudiness, but the 1-day revisiting time and the existing 2 platforms (Aqua and Terra) result are also demanded for MODIS). Compared with the other sensors, the relative 
number of MODIS Ts estimates for snow conditions is smaller, which may be an indication of the MODIS cloud-mask having difficulties to distinguish snow from cloudiness over this specific location. SEVIRI, being a geostationary instrument, provides the largest number of $T s$ estimates given their 15 minutes sampling. Comparing the microwave instruments with AATSR and MODIS, both SSMIS and AMSR-E have a larger number of $T s$ estimates per hour interval as their observations are much less affected by cloudiness. The total number of Ts estimate is larger for SSMIS than for AMSR-E due to the existence of several platforms carrying SSMIS in 2010 (F16, F17, and F18).

Concerning the time of the day, for most agriculture and forest applications one estimate per day should be enough, and the instruments AATSR, AMSR-E (one daytime overpass), MODIS (two), or SSMIS (up to three, linked to the number of orbiting satellites carrying the instrument) could provide the flux estimates. If multiple regular flux estimates per day are required, only the geostationary instrument SEVIRI can provide such sub-daily sampling. Clouds can also severely limit the time sampling for the infrared instruments. The time of the day is also of importance for the polar instruments. If only one flux estimate per day is derived, satellite estimates will be potentially more robust around midday where quantities such as the surface radiation or temperatures have larger values, typically resulting in smaller estimation errors. This can be of importance also when the instantaneous $L E$ estimate is used to derive a daily estimate by assuming that the ratio of $L E$ to some other reference variable (typically the available surface radiative energy) is constant during the day. In this regard MODIS (Aqua) and AMSR-E have more adequate overpass times than AATSR and SSMIS.

\subsection{Satellite surface temperature cross-comparisons}

A comparison of the different satellite $T s$ with the station $T s$ is presented here. Scatter plots of the satellite daytime Ts with respect to the station $T s$ are shown in Figure 4. Notice again the different populations used to derive the statistics, as illustrated by the number of points shown in the scatter plots. The 


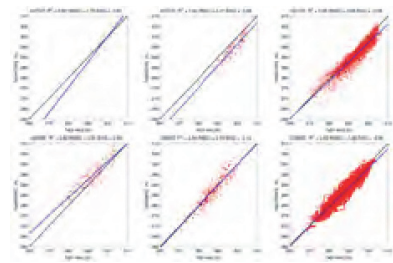

Figure 4: Scatter plots of satellite and observed skin temperature $(T s)$ at the station (from top-left to bottom-middle AATSR, MODIS, SEVIRI, AMSR-E, and SSMIS) and COSMO and observed air temperature $(T a)$ at the station (bottom-right). Plotted the 1:1 line (black) and the linear fit to the data (blue line). Statistics of the difference between the satellite and station $T s$ are given in the plot titles: square of the correlation coefficient $\left(\mathrm{R}^{2}\right)$, mean of the Ts difference (BIAS), and root mean square difference (RMSD).

three infrared instruments AATSR, MODIS, and SEVIRI, have a cold bias with respect to the station $T s$, with AATSR having the smallest bias $(\sim 1 \mathrm{~K}$, but calculated over a very small population compared with MODIS and SEVIRI). Concerning the microwave instruments, SSMIS agrees very well with the station Ts (bias close to zero), while AMSR-E have a relatively large warm bias ( $\sim 4 \mathrm{~K})$. In terms of spatial representativeness, a clear link between satellite footprint and $T s$ differences is not apparent (i.e., that smaller differences are observed for the smaller satellite footprints in principle matching more closely the station surface conditions). In fact, the best agreement in terms of bias and Root Mean Square Difference (RMSD) happens for two sensors having very different resolutions (AATSR, $\sim 1 \mathrm{~km}$, and SSMIS, $\sim 25 \mathrm{~km}$ ).

Statistics of the $T s$ differences (satellite estimate and station) as a function of the time of the day are presented in Figure 5. SEVIRI is the only sensor which makes regular observations throughout the day. The RMSD is slightly smaller for hours around mid-day, compared with the mid-morning hours (even if at mid-day the absolute $T s$ values are larger), but the correlations at mid-day are lower than at mid-morning. This could possibly be explained by the diurnal Ts gradients at this specific location (see Figure 1): the gradients are larger at mid-morning compared with mid-day, which tends to amplify the differences 
(i.e., larger RMSD) but it also helps to increase correlation values (related to the larger Ts variability). Regarding the other sensors, SSMIS is closer than SEVIRI to the station $T s$ for the early-morning overpasses (but SEVIRI is closer for the late-afternoon overpasses), AATSR is closer than MODIS and SEVIRI for the mid-morning overpasses, and SEVIRI is closer than MODIS and AMSRE at mid-day. Notice also that SSMIS agrees better with the station Ts than AMSR-E (with overpasses at different times of the day), and that SSMIS and AMSR-E biases change sign with the time of the day (SSMIS colder than the station $T s$ in the early-morning hours, but AMSR-E and SSMIS warmer than the station $T s$ at mid-day).

Overall, the discrepancies found between the different sensors $T s$ estimates are comparable with the findings from simliar satellite-ground $T s$ comparisons reported elsewhere [e.g. 46, 47, 48, 49]. Identifying the causes of these intersensor differences when compared with a common ground $T s$ estimate is always difficult, but in principle they should be mainly related, on the one hand, to the accuracy and representativeness of the ground longwave radiation based $T s$ estimates, and on the other hand, to issues related to the satellite observations, such as the impact of different viewing geometries, thermal properties of the observed scene, the use of different land surface emissivity estimates in the retrieval, calibration uncertainties of the top-of-atmosphere sensors, and the uncertainties on the parameters used in the retrieval algorithms. It should be noted that differences between the different $T s$ estimates are expected to be closer for the night comparisons due to the more homogeneous heating/cooling conditions of the night scenes.

\subsection{Temperature derived heat fluxes}

The statistics of the comparison of the SEBS heat fluxes (RMSD and square of the correlation, $\mathrm{R}^{2}$ ) with station values are reported in Figure 6. SEBS heat fluxes are derived using the different satellite $T s$ inputs. As reference the absolute RMSD values and averaged heat fluxes (half of the hourly values) are 

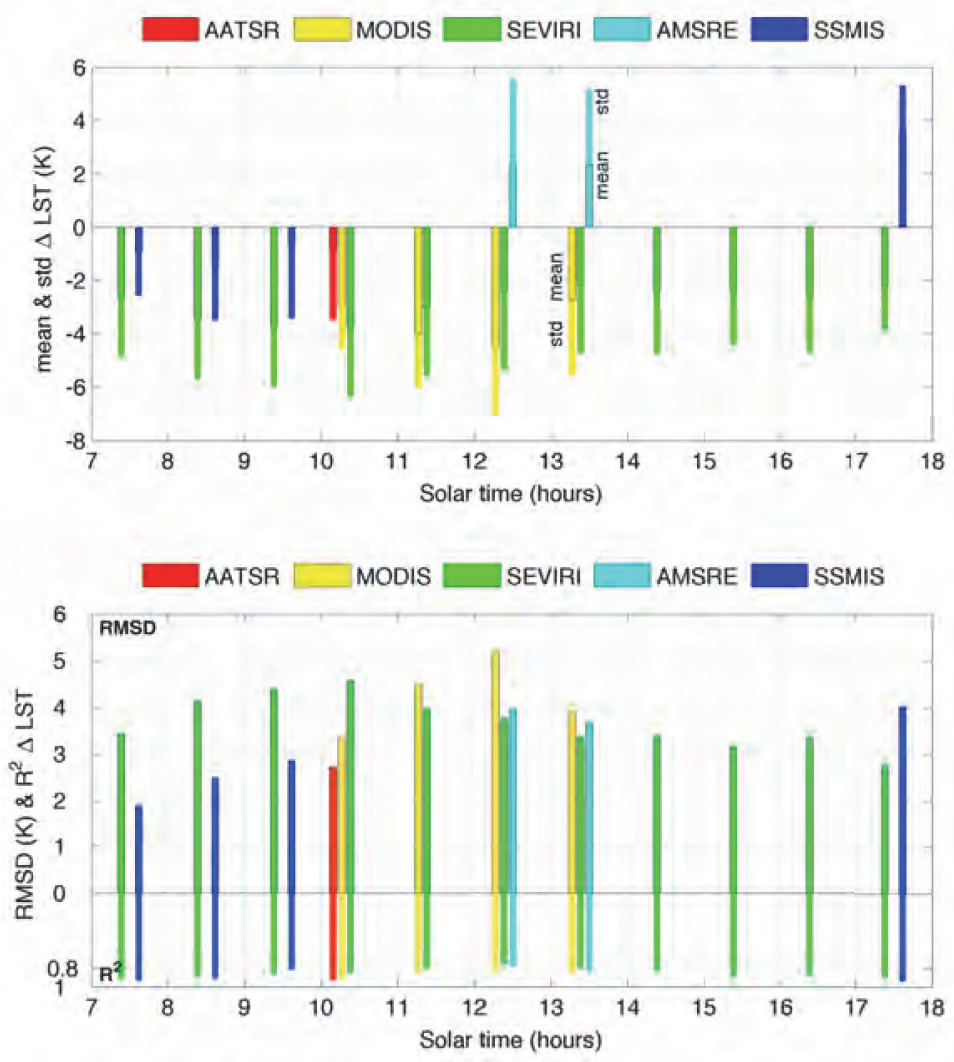

Figure 5: Statistics of the difference between the satellite (AATSR in red, MODIS in yellow, SEVIRI in green, AMSR-E in cyan, and SSMIS in blue) and station skin temperature (Ts) as a function of the local solar time. The top panel shows the mean of the difference (black outlined bar) and the standard deviation of the difference (over-plotted on a bar above the mean bar). The bottom panel shows the root mean square difference (RMSD, positive axis), and the square of the correlation coefficient $\left(\mathrm{R}^{2}\right.$, negative axis). 
RMSD and higher correlations) with the $H$ measured at the station corresponds for most hours to running SEBS with the station Ts. This is expected as the station $T s$ is likely to be a better estimate of the the station temperature, compared with the satellite estimates. For the times of the day when $H$ is small 420 (i.e., early morning and late afternoon), the prediction relative errors are large (even for the fluxes derived with the in situ Ts, e.g., 100 \% RMSD at the 7-8 hour interval). Around mid-day when $H$ is at its maximum the predictions are better $\left(\sim 50 \% \mathrm{RMSD}, \sim 0.7 \mathrm{R}^{2}\right)$. A clear link between satellite and station Ts closeness and the station and satellite $H$ agreement is not apparent. At the 7-11 hour intervals SSMIS compares better with the station $T s$ than SEVIRI, and the SSMIS-derived $H$ correlates better with the station $H$ and have smaller RMSD, compared with SEVIRI, but for the other intervals this relationship is not so obvious (e.g., at the 12-13 hour interval AMSR-E has the largest RMSD comparing with the station $T s$, but the smaller RMSD comparing with the station $H$ ). This suggests that for this specific exercise there is not a clear relationship between $T s$ accuracy (judged by closeness to the station $T s$ ) and the SEBS-derived $H$ accuracy (judged by closeness to the station $H$ ). Different factors are likely to play a role here, including the different spatial scales of the satellite-derived $H$ and the Eddy Covariance observations, the errors in the station $T s$ and $H$ used here as reference, and how the errors in the other inputs required by SEBS impact the produced fluxes for a given $T s$ (e.g., as $H$ depends on the $T s$ - $T a$ difference, there may be combinations of $T s-T a$ that are more likely to represent the "true" temperature difference even if their Ts is not the more accurate).

Figure 6 (bottom panel) shows that the satellite-derived $L E$ can be sometimes slightly closer to the station in terms of RMSD (compared with the $L E$ derived with the in situ Ts). Notice that the hourly statistics for the different estimates are not calculated using the same number of days (see Figure 3), so small difference in RMSD and correlation could be not very representative

445 (i.e., they can just be related to sampling different populations). The relative RMSD and correlations show a better agreement for $L E$ than for $H$. This is 

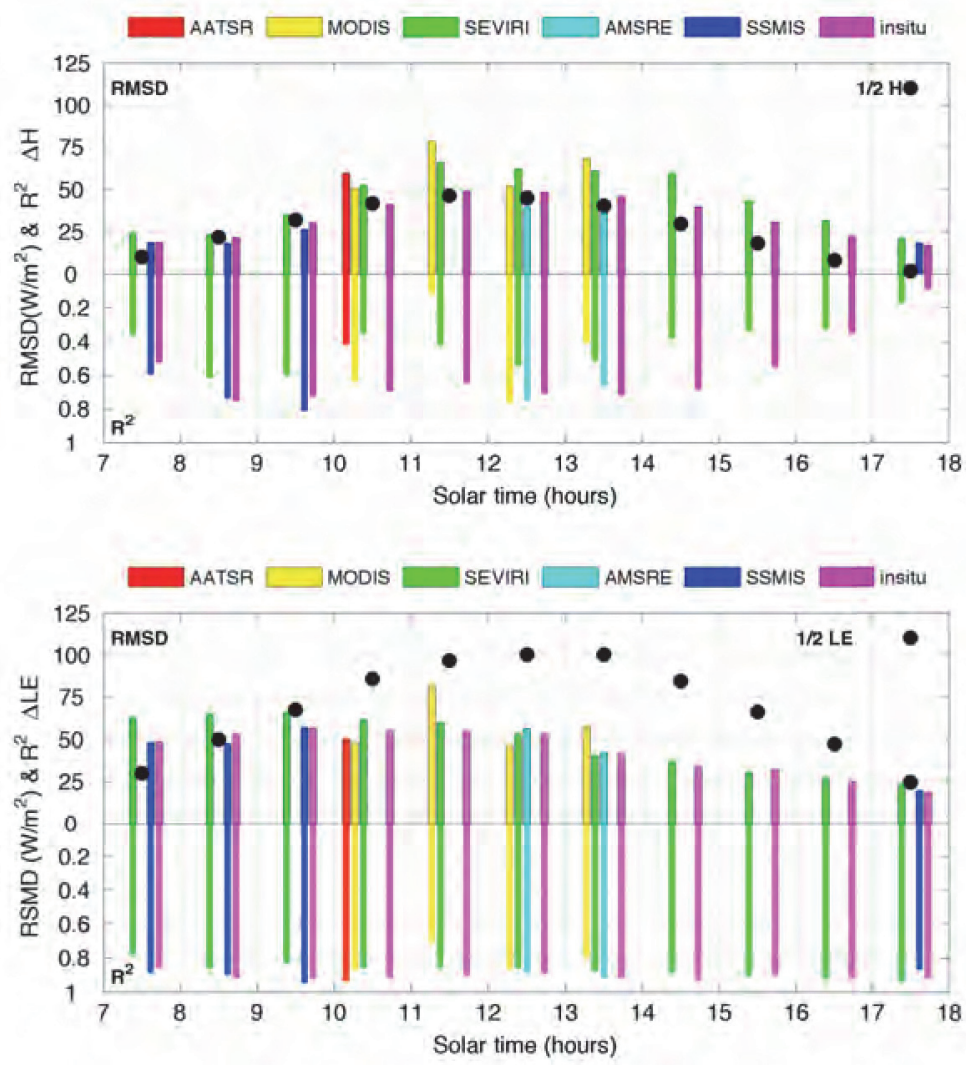

Figure 6: Statistics of the difference between the station Eddy Covariamce (EC) sensible heat flux ( $H$, top panel) and latent heat flux ( $L E$, bottom panel) and the SEBS-derived estimates using skin temperature (Ts) from AATSR (red), MODIS (yellow), SEVIRI (green), AMSR-E (cyan), SSMIS (blue), and the station in situ Ts (magenta), as a function of the local solar time. Displayed are the root mean square difference (RMSD, positive axis), and the square of the correlation coefficient $\left(\mathrm{R}^{2}\right.$, negative axis). Half of the EC hourly-averaged $H$ (top) and $L E$ (bottom) fluxes are displayed as black circles for reference. 
expected given the relatively high evaporative fractions for a large part of the year ( $L E$ comes from the difference between the available energy and $H$, so the impact of errors in $H$ are minimized given that $H$ represents a small fraction of the available energy). The relative RMSD for $L E$ at midday and early afternoon is $\sim 25 \%$ for some of the satellite-derived estimates. Given some of the assumptions (e.g., that fluxes only come from a surface 100\% cover by grass) and the clear miss-match between tower observation fetch and satellite sensor footprint in most cases, this value seems reasonable and compares well with other reported values for this type of vegetation and methodology (e.g., relative daytime RMSD of $\sim 20 \%$ are reported over two grassland sites in [22]). It also compares well with the $15 \%$ to $30 \%$ relative errors typically reported when comparing satellite evaporation and ground measurements over different landscapes $[13,3]$, which gives an idea of the present accuracy available to infer evaporation for agriculture and forest applications.

\section{Conclusions}

Surface heat fluxes are an essential variable to monitor crops and forest status. Their estimation by remote sensing methodologies provides the possibility to routinely analyze large areas, which is of importance to manage cropland and forested areas given the typically limited number of ground observations at most locations. This paper contributes by demonstrating the production of remotely sensed land surface temperature $(T s)$-driven surface heat fluxes at a grassland site situated at the Rietholzbach Research Catchment in northeastern Switzerland.

The exercise has been conducted by running a specific heat flux methodology (SEBS) driven by a number of satellite derived $T s$ estimates (from the instruments AATSR, MODIS, SEVIRI, AMSR-E, and SSMIS). The Ts estimates have been compared with a ground estimate derived from the broadband longwave radiometric observations at the station, and the satellite heat flux es- 
other previous site studies, the heat fluxes are estimated from sensors operating at different resolutions (from $\sim 1$ to $\sim 25 \mathrm{~km}$ ), time sampling (from twice a day to half hourly), and wavelengths (infrared and microwaves), and two types of ground observations of $L E$ (Eddy Covariance (EC) flux measurements and evaporation estimates from a lysimeter) are available to evaluate the satellite estimates.

The main conclusions of the study can be summarized as:

- Satellite $T s$ estimates that can be used for heat flux estimation exist at different spatial resolutions and time sampling during the day. The infrared estimates are limited in terms of temporal coverage (only clear-sky estimates), while the microwave estimates are all-sky but are limited in terms of spatial resolution (one order of magnitude coarser than the infrarred estimates). Satellite overpass can also be an issue for the sensors in polar orbits (i.e., only two estimates per day), although most agriculture and forest applications do not require sub-daily time resolution. In principle sensors with overpasses at midday (e.g., MODIS and AMSR-E in Terra) are more adequate than sensors with early morning and late afternoon overpasses (e.g., SSMIS) as the typically larger fluxes at midday facilitate the flux estimation.

- The satellite Ts estimates can be compared with the station ground estimates, but the comparison suffers from spatial miss-match even for the highest infrared satellite estimates (e.g., MODIS footprint $\sim 10$ times larger than the footprint of the longwave radiometric observations at the station). The different nature of the observations (e.g., station broadband radiometers 2 meters above the ground versus satellite narrow-band radiometers observing at a given angle from space) also plays a role in the differences observed. Root Mean Square Differences (RMSDs) of the daytime satellite and station $T s$ are 2.72 (AATSR), 4.41 (MODIS), 3.59 (SEVIRI), 3.81 (AMSR-E), and 2.79 (SSMIS), but given the difficulties mentioned in the comparison it is not possible to use the agreement with 
the station $T s$ as an absolute metric to judge satellite $T s$ accuracy over this specific location.

- The comparison of the satellite $T s$-derived heat fluxes with the station EC is also challenging. Again, differences between the area integrated in the satellite flux measurements and the tower observations make the comparison difficult. Also, discrepancies are observed when the ground EC fluxes measured at the station are compared with some other measurements at the site (e.g., disagreements between the EC and lysimeter $L E$, or between the sum of the observed EC $L E$ and $H$ and the available energy given by $R n-G)$. This suggests that the EC fluxes, although very valuable to evaluate the $T s$-derived fluxes, cannot be considered as an absolute reference.

- For those sensors with midday overpasses, a RMSD of $\sim 25 \%$ are found when comparing the instantaneous $L E$ at satellite overpass with the EC observations. Larger differences with the EC observations are found for $H$ (best RMSD values of $\sim 50 \%$ at midday for some $T s$ estimates). The RMSD of $\sim 25 \%$ compares well with the $15 \%$ to $30 \%$ relative errors typically reported when comparing satellite evaporation and ground measurements over different landscapes $[13,3]$, and gives an idea of the present accuracy available to infer evaporation for agriculture and forest applications.

- Although in principle more accurate $T s$ estimates will necessarily result in more accurate heat flux estimations, a clear link between $T s$ and $L E$ accuracy is not apparent for this specific comparison. This can be the result of the quality of the $T s$ and $L E$ metrics used for the evaluation (closeness to the station ground estimates), but it can also be linked to SEBS accuracy not only depending on $T s$ but on other variables (notably $T a$ and $R n$ for this specific location where relatively constant vegetation conditions should not make a large contribution to flux changes). 

framework of the European Space Agency (ESA) Data User Element (DUE) GlobTemperature project (http://data.globtemperature.info), which is managed by ESA technical officer Simon Pinnock. It has also been supported by the ESA Support To Science Element (STSE) WACMOS-ET project (http:

Case Study component of the GlobTemperature project and Darren Ghent as project scientist for the AATSR data. The AATSR data have been obtained from the GlobTemperature data portal (http://data.globtemperature.info/). The MODIS data were obtained from the LP DAAC, operated by the U.S. 
Geological Survey (USGS) and the National Aeronautics and Space Administration (NASA). The SEVIRI/MSG surface temperature data were generated by the Land Surface Analysis Satellite Applications Facility (LSA SAF, http://landsaf.ipma.pt funded by EUMETSAT. The SSMIS observations have been provided by the Satellite Application Facility on Climate Monitoring (CMSAF, http://www.cmsaf.eu/), funded by EUMETSAT. The AMSR-E radiances were provided by the National Snow and Ice Data Center (NSIDC, https: //nsidc.org/). A training database for the AMSRE inversions was provided by the Atmospheric and Environmental Research, Inc., Lexington, Massachusetts, USA, and generated upon work supported by the NASA under contract NNH04CC43C.

[1] A. K. Betts, Land-surface-atmosphere coupling in observations and models, Journal of Advances in Modeling Earth Systems 2 (2009) 4-18.

[2] S. Sorooshian, R. Lawford, P. Try, Water and energy cycles: Investigating the links, WMO Bulletin 54 (2005) 58-64.

[3] K. Wang, R. E. Dickinson, A review of global terrestrial evapotranspiration: Observation, modeling, climatology, and climatic variability, Reviews of Geophysics 50 (2) (2012) RG2005-54.

[4] S. M. Dunn, R. Mackay, Spatial variation in evapotranspiration and the influence of land use on catchment hydrology, Journal of Hydrology 171 (14) (1995) 49-73.

[5] D. C. Le Maitre, D. B. Versfeld, Forest evaporation models: relationships between stand growth and evaporation, Journal of Hydrology 193 (1) (1997) $240-257$.

[6] P. H. Gowda, J. L. Chavez, P. D. Colaizzi, S. R. Evett, T. A. Howell, J. A. Tolk, Et mapping for agricultural water management: present status and challenges, Irrigation Science 26 (3) (2008) 223-236. doi:10.1007/ s00271-007-0088-6. 
[7] V. R. N. Pauwels, W. Timmermans, A. Loew, Comparison of the estimated water and energy budgets of a large winter wheat field during AgriSAR 2006 by multiple sensors and models, Journal of Hydrology 349 (3-4) (2008) 425440.

[8] D. Baldocchi, E. Falge, L. Gu, R. Olson, D. Hollinger, S. Running, P. Anthoni, C. Bernhofer, K. Davis, R. Evans, J. Fuentes, A. Goldstein, G. Katul, B. Law, X. Lee, Y. MALHI, T. Meyers, W. Munger, W. Oechel, K. T. Paw, K. Pilegaard, H. P. Schmid, R. Valentini, S. Verma, T. Vesala, K. Wilson, S. Wofsy, FLUXNET: A New Tool to Study the Temporal and Spatial Variability of Ecosystem-Scale Carbon Dioxide, Water Vapor, and Energy Flux Densities., Bulletin of the American Meteorological Society 82 (1) (2001) 2415-2434.

[9] J. Monteith, Evaporation and environment, Symp. Soc. Exp. Biol 19 (20523) (1965) 4.

[10] C. Priestley, R. Taylor, On the assessment of surface heat flux and evaporation using large-scale parameters, Mon. Weather Rev, Mon. Weather Rev. 100 (1972) 81-92.

[11] R. G. Allen, L. S. Pereira, D. Raes, M. Smith, FAO Irrigation and drainage paper No. 56, Rome: Food and Agriculture Organization of ..., 1998.

[12] J. D. Tarpley, Monthly Evapotranspiration from Satellite and Conventional Meteorological Observations, Journal of Climate 7 (1994) 704-713.

[13] J. D. Kalma, T. R. McVicar, M. F. McCabe, Estimating Land Surface Evaporation: A Review of Methods Using Remotely Sensed Surface Temperature Data, Surveys in Geophysics 29 (4-5) (2008) 421-469.

[14] W. P. Kustas, K. S. Humes, Spatially Distributed Sensible Heat Flux over a Semiarid Watershed. Part II: Use of a Variable Resistance Approach with Radiometric Surface Temperatures, Journal of Applied Meteorology 36 (1997) 293-311. 
[15] W. P. Kustas, J. M. Norman, Evaluating the Effects of Subpixel Heterogeneity on Pixel Average Fluxes, Remote Sensing of Environment 74 (3) (2000) 327-342.

[16] K. Nishida, An operational remote sensing algorithm of land surface evaporation, Journal of Geophysical Research 108 (D9) (2003) 4270-14.

[17] J. M. Norman, M. C. Anderson, W. P. Kustas, A. N. French, J. Mecikalski, R. Torn, G. R. Diak, T. J. Schmugge, B. C. W. Tanner, Remote sensing of surface energy fluxes at 101-m pixel resolutions, Water Resources Research 39 (8) (2003) 1221.

[18] M. C. Anderson, J. M. Norman, J. R. Mecikalski, J. A. Otkin, W. P. Kustas, A climatological study of evapotranspiration and moisture stress across the continental United States based on thermal remote sensing: 1. Model formulation, Journal of Geophysical Research 112 (D10) (2007) D10117-17.

[19] Z. Su, The Surface Energy Balance System (SEBS) for estimation of turbulent heat fluxes, Hydrology and Earth System Sciences 6 (1) (2002) 85-100.

[20] T. N. Carlson, W. J. Capehart, R. R. Gillies, A new look at the simplified method for remote sensing of daily evapotranspiration, Remote Sensing of Environment 54 (2) (1995) 161-167.

[21] L. Jia, Z. Su, B. van den Hurk, M. Menenti, A. Moene, H. A. R. De Bruin, J. J. B. Yrisarry, M. Ibanez, A. Cuesta, Estimation of sensible heat flux using the Surface Energy Balance System (SEBS) and ATSR measurements, Physics and Chemistry of the Earth, Parts A/B/C 28 (1-3) (2003) 75-88.

[22] H. Su, E. F. Wood, M. F. McCabe, Z. Su, Evaluation of remotely sensed evapotranspiration over the CEOP EOP-1 reference sites, Journal of the Meteorological Society of Japan 85A (2007) 439-459.

[23] M. F. McCabe, E. F. Wood, Scale influences on the remote estimation of evapotranspiration using multiple satellite sensors, Remote Sensing of Environment 105 (4) (2006) 271-285. 
[24] J. Lu, Z.-L. Li, R. Tang, B.-H. Tang, H. Wu, F. Yang, J. Labed, G. Zhou, Evaluating the SEBS-estimated evaporative fraction from MODIS data for a complex underlying surface, Hydrological Processes (2012) 1-11.

[25] Y. Wang, X. Li, S. Tang, Validation of the SEBS-derived sensible heat for FY3A/VIRR and TERRA/MODIS over an alpine grass region using LAS measurements, International Journal of Applied Earth Observations and Geoinformation 23 (2013) 226-233.

[26] A. Ershadi, M. F. McCabe, J. P. Evans, N. W. Chaney, E. F. Wood, Multi-site evaluation of terrestrial evaporation models using FLUXNET data, Agricultural and Forest Meteorology 187 (2014) 46-61.

[27] S. I. Seneviratne, I. Lehner, J. Gurtz, A. J. Teuling, H. Lang, U. Moser, D. Grebner, L. Menzel, K. Schroff, T. Vitvar, M. Zappa, Swiss prealpine Rietholzbach research catchment and lysimeter: 32 year time series and 2003 drought event, Water Resources Research 48 (6) (2012) W06526.

[28] M. Hirschi, D. Michel, I. Lehner, S. I. Seneviratne, A site-level comparison of lysimeter and eddy-covariance flux measurements of evapotranspiration, Hydrology and Earth System Sciences Discussions (2016) 1-34.

[29] A. Monin, A. Obukhov, Basic laws of turbulent mixing in the surface layer of the atmosphere, Tr. Akad. Nauk SSSR Geophiz. Inst. 24 (151) (1954) $163-187$.

[30] D. Ghent, Land Surface Temperature Validation and Algorithm Verification (Report to European Space Agency), ESA Contract Number: 19054/05/NL/FF, University of Leicester, NILU.

[31] I. F. Trigo, C. C. Dacamara, P. Viterbo, J.-L. Roujean, F. Olesen, C. Barroso, F. Camacho-de Coca, D. Carrer, S. C. Freitas, J. García-Haro, B. Geiger, F. Gellens-Meulenberghs, N. Ghilain, J. Meliá, L. Pessanha, N. Siljamo, A. Arboleda, The Satellite Application Facility for Land Sur- 
face Analysis, International Journal of Remote Sensing 32 (10) (2011) 27252744 .

[32] W. Wang, S. Liang, T. Meyers, Validating MODIS land surface temperature products using long-term nighttime ground measurements, Remote Sensing of Environment 112 (3) (2008) 623-635.

[33] K. Fennig, A. Andersson, M. Schrder, Fundamental Climate Data Record of SSM/I / SSMIS Brightness Temperatures. Satellite Application Facility on Climate Monitoring. (2015). doi:DOI:10.5676/EUM \_SAF\_CM/FCDR_ MWI/V002.

URL http://dx.doi.org/10.5676/EUM_SAF_CM/FCDR_MWI/V002

[34] P. Shcroft, F. J. Wentz, AMSR-E/Aqua L2A Global Swath SpatiallyResampled Brightness Temperatures, Version 3 (2013). doi:doi:http: //dx.doi.org/10.5067/AMSR-E/AE\$\_L2A.003.

[35] C. Prigent, C. Jimenez, F. Aires, Toward "all weather," long record, and real-time land surface temperature retrievals from microwave satellite observations, Journal of Geophysical Research: Atmospheres 121 (10) (2016) $5699-5717$.

[36] F. Aires, C. Prigent, W. Rossow, M. Rothstein, A new neural network approach including first-guess for retrieval of atmospheric water vapor, cloud liquid water path, surface temperature and emissivities over land from satellite microwave observations, Journal of Geophysical Research 106 (2001) $14887-14907$.

[37] J.-L. Moncet, P. Liang, J. F. Galantowicz, A. E. Lipton, G. Uymin, C. Prigent, C. Grassotti, Land surface microwave emissivities derived from AMSR-E and MODIS measurements with advanced quality control, Journal of Geophysical Research 116 (D16) (2011) D16104-20.

[38] S. W. Seemann, E. E. Borbas, R. O. Knuteson, G. R. Stephenson, H.L. Huang, Development of a Global Infrared Land Surface Emissivity 
Database for Application to Clear Sky Sounding Retrievals from Multispectral Satellite Radiance Measurements, Journal of Applied Meteorology and Climatology 47 (1) (2008) 108-123.

[39] K. Wang, Estimation of surface long wave radiation and broadband emissivity using Moderate Resolution Imaging Spectroradiometer (MODIS) land surface temperature/emissivity products, Journal of Geophysical Research 110 (D11) (2005) D11109-13.

[40] P. Stackhouse, S. Gupta, S. Cox, J. Mikovitz, T. Zhang, M. Chiacchio, 12-year surface radiation budget data set, GEWEX News 14 (4) (2004) $10-12$.

[41] C. R. Ferguson, E. F. Wood, An Evaluation of Satellite Remote Sensing Data Products for Land Surface Hydrology: Atmospheric Infrared Sounder*, Journal of Hydrometeorology.

[42] T. D. Mitchell, P. D. Jones, An improved method of constructing a database of monthly climate observations and associated high-resolution grids, International Journal of Climatology 25 (6) (2005) 693-712.

[43] D. Michel, C. Jimenez, D. G. Miralles, M. Jung, M. Hirschi, A. Ershadi, B. Martens, M. F. McCabe, J. B. Fisher, Q. MU, S. I. Seneviratne, E. F. Wood, D. Fernández-Prieto, The WACMOS-ET project \&ndash; Part 1: Tower-scale evaluation of four remote-sensing-based evapotranspiration algorithms, Hydrology and Earth System Sciences 20 (2) (2016) 803-822.

[44] J. van der Kwast, W. Timmermans, A. Gieske, Z. Su, A. Olioso, L. Jia, J. Elbers, D. Karssenberg, S. de Jong, Evaluation of the Surface Energy Balance System (SEBS) applied to ASTER imagery with fluxmeasurements at the SPARC 2004 site (Barrax, Spain), Hydrology and Earth System Sciences 13 (7) (2009) 1337-1347.

[45] H. Hoffmann, H. Nieto, R. Jensen, R. Guzinski, P. Zarco-Tejada, T. Friborg, Estimating evaporation with thermal UAV data and two-source en- 
ergy balance models, Hydrology and Earth System Sciences 20 (2) (2016) $697-713$.

[46] G. Sòria, J. A. Sobrino, ENVISAT/AATSR derived land surface temperature over a heterogeneous region, Remote Sensing of Environment 111 (4) (2007) 409-422.

[47] I. F. Trigo, I. T. Monteiro, F. Olesen, E. Kabsch, An assessment of remotely sensed land surface temperature, Journal of Geophysical Research: Atmospheres 113 (D) (2008) D17108.

[48] J. Catherinot, C. Prigent, R. Maurer, F. Papa, C. Jimenez, F. Aires, W. B. Rossow, Evaluation of "all weather" microwave-derived land surface temperatures with in situ CEOP measurements, Journal of Geophysical Research: Atmospheres 116 (D) (2011) D23105.

[49] W. Yu, M. Ma, X. Wang, L. Geng, J. Tan, J. Shi, Evaluation of MODIS LST Products Using Longwave Radiation Ground Measurements in the Northern Arid Region of China, Remote Sensing 6 (11) (2014) 11494-11517. 